\title{
Performance and Stability of Dedicated Aberration-Corrected STEMs: a User's Perspective
}

\author{
D.M. Kepaptsoglou ${ }^{1}$, A.R. Lupini ${ }^{2}$, D. Mücke-Herzberg ${ }^{1}$, G. Vaughan ${ }^{1}$ and Q.M. Ramasse ${ }^{1}$ \\ ${ }^{1}$ SuperSTEM Laboratory, STFC Daresbury Campus, Daresbury, United Kingdom \\ ${ }^{2}$ Materials Science \& Technology Division, Oak Ridge National Laboratory, Oak Ridge, TN, U.S.A.
}

As the feature list of modern aberration-corrected electron microscopes grows longer, the requirements for ultimate stability, be-it of individual components of the instruments or of the dedicated controlled environments they are installed in, become stricter and stricter [1-3]. Yet, even the best designed room environments fail or slowly go out of specifications, and rare are the research groups who can afford the time to strictly monitor every environmental or instrumental parameter [4]. Ironically however, modern instruments have become so powerful that there is a risk that instabilities go unnoticed once a machine has left the factory or the prying eyes of the installation engineer, until they become truly crippling. It would therefore be instructive to monitor how real-life parameters influence the day-to-day operations of state-of-the-art aberration-corrected instruments and compare data across laboratories operating similar equipment, or even between similar instruments installed on the same site but in rooms with different designs.

This study will attempt to compare the long term stability of aberration coefficients, as measured by the manufacturer-provided diagnosis software, of similar dedicated aberration-corrected scanning transmission electron microscopes installed within two different operating environments. We will attempt to evaluate the effect of a number of environmental parameters, often referred to as 'site stability' including temperature control, cooling water temperature and flow, local magnetic fields and site vibrations. In addition, we will try to correlate 'lab practices' with the long term performance of our instruments, for example by comparing cycles in high tension voltages (as the users go up and down the range of operating acceleration voltages), number of sample exchanges over a number of months, 'remote' (i.e. separate operator room) vs local operation and compare the influence of different 'best practice' approach at the two sites.

The report will in no way attempt to pinpoint weaknesses (or strengths) in a particular manufacturer design, but rather aims at providing a user's perspective on what level of stability one can expect in a 'real-laboratory' environment, and whether any measured instability really affects what after all matters most to users of advanced scientific instrumentation: their productivity and scientific output.

\section{References}

[1] D. C. Bell, C. J. Russo, and G. Benner, Microsc Microanal 16, 386 (2010).

[2] A. Muller and J. Grazul, J Electron Microsc 50 , 219 (2001).

[3] D. A. Muller, E. J. Kirkland, M. G. Thomas et al., Ultramicroscopy 106, 1033 (2006).

[4] J. Barthel and A. Thust, Ultramicroscopy 134, 6 (2013).

[5] SuperSTEM is the UK National Facility of aberration corrected STEM, funded by the EPSRC 
a)

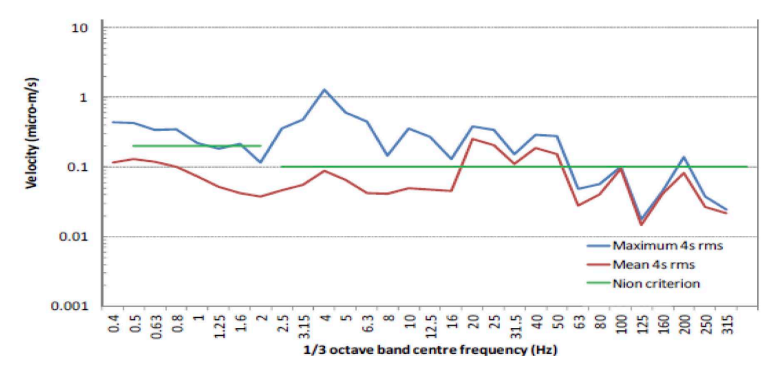

b)

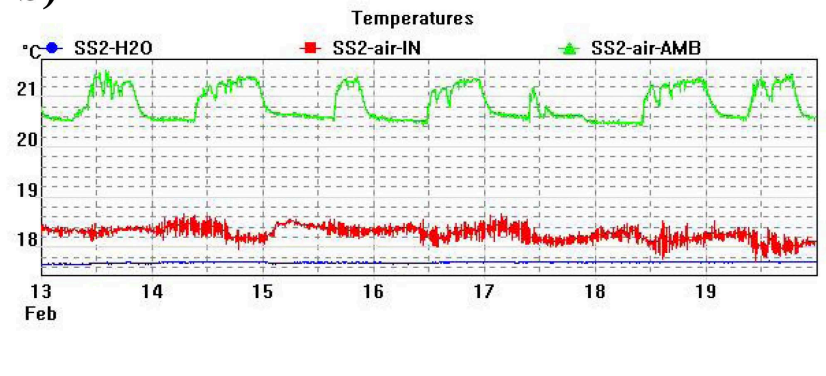

Figure 1: a) Ambient vibration measurements of the SuperSTEM building and site, b) ambient and cooling water temperature monitoring of the Nion UltraSTEM ${ }^{\mathrm{TM}} 100$ instrument installed at SuperSTEM laboratory, Daresbury, UK. Although no perfect, these conditions allow for a very smooth running of the instrument: the goal of this study is to assess the exact impact on optical parameters and long term instrument stability of small deviations from ideal environmental conditions will.

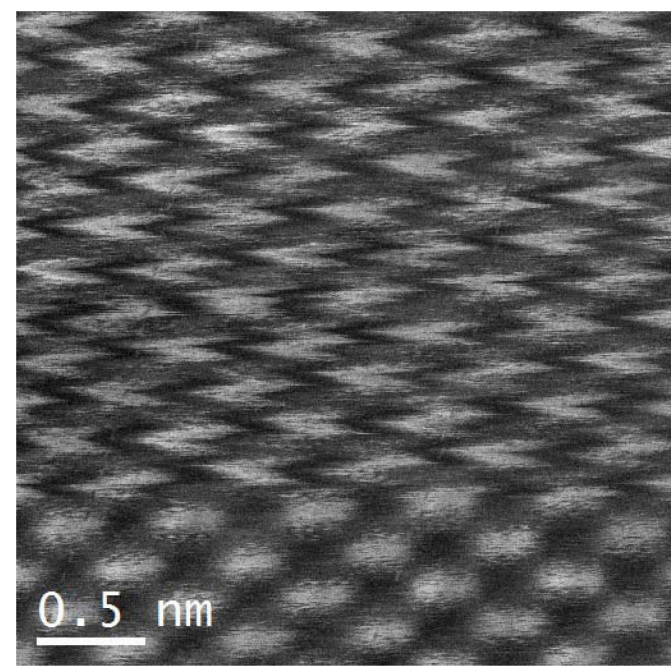

Figure 2: HAADF STEM image, showing an apparent immediate recovery of imaging conditions after electromagnetic field spike due to spot welding taking place above the microscope room, during building works at the SuperSTEM site in August 2013. Although the conditions for atomic-resolution imaging seem to recover immediately after the event, the disturbance had a measurable impact on optical parameters and their stability during the rest of the day. 